\title{
Effect of transported hospital resources on neurologic outcome after out-of-hospital cardiac arrest
}

\author{
JOO YEONG KIM ${ }^{1}$, SUNGWOO MOON 1 , JONG HAK PARK 1 , HAN JIN CHO ${ }^{1}$, JU HYUN SONG ${ }^{1}$, \\ WOOCHAN JEON ${ }^{2}$, HANSOEK CHANG ${ }^{3}$, YOUNG SUN RO ${ }^{4}$, SANG DO SHIN ${ }^{5}$ \\ 1 Department of Emergency Medicine, Korea University Ansan Hospital, Ansan, Gyeonggi-do, South Korea \\ 2 Department of Emergency Medicine, Inje University Ilsanpaik Hospital, Goyang-si, Gyeonggi-do, South Korea \\ 3 EMS Information and Planning Team, National Emergency Medical Center, Seoul, South Korea \\ 4 Department of Emergency Medicine, Seoul National University Biomedical Research Institute, 101 Daehak-Ro, Jongno-Gu, Seoul 110-744, South Korea \\ 5 Department of Emergency Medicine, Seoul National University College of Medicine, Seoul, South Korea
}

Corresponding author

Sungwoo Moon

Department of Emergency Medicine

Korea University Ansan Hospital

Ansan, Gyeonggi-do, South Korea

Phone: +82-31-412-4994

Fax: $+82-31-412-4273$

E-mail:yg9912@korea.ac.kr

\section{ABSTRACT}

Objective. Appropriate regional transport protocol for out-of-hospital cardiac arrest (OHCA) patients is important for achieving favorable outcomes in a certain community. This study aimed to investigate the effect of transported hospital resources on the neurologic outcome after OHCA.

Methods. We categorized cardiac receiving centers (CRC) in our community into two levels (primary [P-CRC] and definite CRC [D-CRC]) according to the hospital resources that were identified by the Hospital Assessment Survey in 2015. OHCA patients with presumed cardiac etiology resuscitated by emergency medical service providers between 2012 and 2014, were enrolled in the study. The main exposure was the level of CRC. The primary endpoint was discharge with good neurologic outcomes. We compared outcomes between CRCs after adjusting for potential confounders.

Results. Among the 9,912 patients, 5,876 were transported to P-CRC and 4,036 to D-CRC from 2012 to 2014. Patients admitted to D-CRC showed better neurologic outcome than those admitted to P-CRC $(6.2 \%$ vs $1.5 \%, \mathrm{p}<0.001)$. With regard to patients who survived to admission, the neurologic outcome of patients in D-CRC was better than those in P-CRC $(11.3 \%$ vs $3.3 \%, \mathrm{p}<0.001)$. In the multivariable logistic model, the adjusted odds ratio for all OHCA patients was 2.10 (95\% confidence interval, 1.51-2.95).
Conclusion. Transportation of OHCA patients to the D-CRC resulted in significantly good neurologic outcome than those transported to P-CRC. Further research is needed to establish a regional OHCA transport protocol.

Key words: cardiac arrest, outcome, regionalization

\section{INTRODUCTION}

Out-of-hospital cardiac arrest (OHCA) is the leading cause of morbidity and mortality. (1) Although much effort has been devoted, the outcome is still poor in Korea, with an $8.0 \%$ survival rate and only $5.8 \%$ with good neurologic outcomes. (2)

In 2006, integrated post-arrest care was added to the chain of survival, emphasizing the importance of comprehensive multidisciplinary post-arrest care. Currently, an increasing number of publications have reported on the beneficial effects of targeted temperature management and other forms of post-resuscitation care on neurologic outcomes. (3-5)

However, not all cardiac arrest receiving facilities have the capacity to provide comprehensive post-arrest care. To overcome this issue, the concept of 'cardiac resuscitation center' or 'regionalization strategy of OHCA care' has emerged. In 2010, the American Heart Association (AHA) proposed two levels of cardiac resuscitation centers and suggested the criteria for each level of CRC. (6) The main recommended strategy is to transfer patients with spontaneous circulation after OHCA to specialized CRCs for comprehensive care. (6) Locally, in Los Angeles, OHCA patients with initial shockable rhythm were sent to STelevation myocardial infarction (STEMI) receiving centers, which resulted in higher rates of neurologically intact survival. (7) In Arizona, the implementation of a statewide system of cardiac receiving centers resulted in the improvement of survival in patients with intact neurologic status. (8) The appropriate transport strategy for cardiac arrest patients in a certain community could be designed and implemented after the assessment of hospital resources and performances. In this study, we aimed to categorize CRCs according to the hospital resources and identified outcome differences among facilities in our community.

\section{MATERIALS AND METHODS}

\section{Study design and setting}

This retrospective observational study was conducted using data from the Hospital Assessment Survey and national OHCA database. A survey on the availability of resources, number of personnel, and physical infrastructures of each emergency medical center in the Gyeonggi province was conducted in 2015 by the Gyeonggi Emergency Support Center. The Gyeonggi Emergency Support Center is a regional center under 
the National Emergency Medical Center, which serves as the central emergency medical service (EMS) control tower. The survey was conducted primarily via the $\mathrm{Na}$ tional Emergency Department Information System (NEDIS) and additively by email to all emergency departments (EDs) that provided care to at least one OHCA patient who was transported to the said unit between 2013 and 2014.

Gyeonggi province has an area of 10,175 $\mathrm{km} 2$, which is seventeen times larger than the Seoul Metropolitan Area and nine times larger than Hong Kong. It occupies $10.1 \%$ of the national territory and is the 5 th largest province in Korea. The population in Gyeonggi almost reached a total of 12.5 million, which is almost a quarter of the total Korean population.

The Korean EMS system is managed by the fire department and provided by the government. It offers single-tiered basicto-intermediate ambulance services. The ambulance crew can perform cardiopulmonary resuscitation (CPR) at the scene and during transport, with performances comparable to the intermediate emergency medical technician (EMT) level in the United States, including intravenous catheterization and advanced airway under direct medical oversight. Advanced circulatory life support, including administration of drugs such as epinephrine, is not legally authorized in the field in most areas, and EMTs cannot declare death or stop CPR without direct medical oversight. They call for medical directions if OHCA victims show definite signs of death such as decapitation, rigor mortis, decomposition, or dependent lividity and withdraw resuscitation. All OHCA patients with resuscitation attempts are transported to the nearest ED according to the EMS standard procedure protocol. There are 218 ambulances operating in Gyeonggi province, and usually, two or three EMTs ride in each ambulance.

\section{Study population}

EMS-treated OHCA patients with a presumed cardiac etiology transported by Gyeonggi EMS to hospitals located in Gyeonggi province were included in this study. Among them, OHCA patients, whose final outcomes are available on medical records during 2012-2014, were finally enrolled in the analysis. Patients with presumed cardiac etiology were defined if they had no definite evidence of non-cardiac causes. (9) EMS-treated OHCA patients were defined if they received at least one of the following resuscitation efforts: chest compression, rescue breathing, and defibrillation.

\section{Main exposure and variables}

The main exposure was the classification of CRCs, which was attained using the results of the Gyeonggi Hospital Assessment Survey. Facilities were classified as definite CRC (D-CRC) if they had a standardized resuscitation protocol in the $\mathrm{ED}$, admitted OHCA patients who achieved return of spontaneous circulation (ROSC), had a standardized therapeutic temperature management (TTM) protocol, if they offered percutaneous coronary intervention (PCI) 24 hours a day and 7 days a week, if they had a cardiac arrest registry for quality assurance, could confirm brain death, had rehabilitation programs for OHCA survivors, and offered community-based resuscitation training programs. Other facilities were defined as primary CRC (P-CRC). Classification of CRC was based on the internationally accepted criteria $(10,11)$ and consensus from expert meetings.

Data of potential confounders such as age; gender; pre-arrest medical conditions such as cardiac disease, hypertension and diabetes mellitus; initial ECG rhythm at scene; the presence of witnesses; provision of bystander CPR; EMS response interval; EMS scene resuscitation interval; patient transport interval; and pre-hospital ROSC were obtained from the national OHCA database.

\section{Data setting}

Pre-hospital and hospital data of OHCA patients transported to hospitals were collected using the national OHCA database in Korea. The national OHCA database captures all incident cases of OHCA in the country using the EMS run sheet for basic ambulance operation information and the national OHCA registry for hospital care and survival outcomes via hospital medical record review. The EMS run sheet and EMS cardiac arrest registry are abstracted from the EMS database of the National Emergency Management Agency (NEMA), and a subsequent medical record review is conducted by reviewing the medical records of each OHCA patient transferred to a hospital by trained medical record reviewers from the Korea Centers for Disease Control and Prevention (CDC). Monthly quality assurance meetings are held by the Korea CDC Data Quality Control team, which consists of emergency physicians, epidemiologists, statistical experts, representatives from NEMA, and medical record reviewers.

\section{Outcome Measures}

The primary outcome was good neurologic recovery classified according to cerebral performance category (CPC) and defined as favorable if the CPC was 1 or 2 . The secondary outcome was survival to discharge. All outcome measures were based on a review of medical records.

\section{Statistical Analysis}

Descriptive statistics between CRCs for categorical variables are presented as frequency distributions and percentages. Continuous variables are reported as medians and interquartile ranges (IQRs). Univariate comparisons of the distributions of demographic and clinical factors were analyzed using the chi-square test for discrete variables and the Wilcoxon rank-sum test for continuous variables. We compared the outcomes between CRCs using crude and adjusted odds ratios (aORs) and 95\% confidence intervals (CIs). Multivariable logistic regression was performed after adjusting for potential confounders including Utstein co-variables (age, sex, witness status, location of arrest [public vs private], bystander CPR, EMS response time, EMS transport time and pre-hospital electrocardiogram pattern [shockable vs non-shockable]), pre-existing comorbidities (hypertension, diabetes, and cardiac disease), and result of pre-hospital ROSC.

We also analyzed for collinearity and tested if variables had conditional index $>30$ and variance decomposition proportion $>0.5$. No multicollinearity was detected in our models and all terms were retained. All data were analyzed using SAS 9.4 (SAS Institute Inc., NC, USA).

The study protocol was approved by the Korea University Ansan Hospital Institutional Review Board to be conducted with a waiver of informed consent (IRB number: K2018-0500-001).

\section{RESULTS}

Among a total of 14,951 EMS-treated OHCA patients, 4,126 were definitely of non-cardiac etiology, 329 were transported to hospitals in other regions, and the final outcomes of 584 were unavailable. A total of 9,912 cases were enrolled in the study (Figure 1).

Of the 90 cardiac arrest receiving facilities, 77 facilities responded to the survey. All $13(13.1 \%)$ facilities that did not respond to the survey were non-ED facilities. Of the 77 cardiac arrest receiving facilities, 50 
facilities (64.9\%) admitted post-ROSC (return of spontaneous circulation) patients and 22 facilities (28.6\%) were available to provide therapeutic hypothermia. A total of $29(37.7 \%)$ facilities offered 24/7 PCI, and $27(35.1 \%)$ provided a quality management program on OHCA resuscitation and treatment. The facility was classified as D-CRC if they had a standard resuscitation protocol at the ED; had the device, manpower, and standard protocol for TTM; if PCI was available 24/7; if they had an OHCA registry system; if they could confirm brain death, had an available rehabilitation program for post-resuscitated patients, and provided community-based CPR programs (Table 1).

Among 9,912 OHCA, 4,036 patients $(40.7 \%)$ were initially transported to DCRC. The proportion of pre-existing Comorbidities was higher among OHCA patients transported to D-CRC. OHCA patients transported to D-CRC showed better neurologic outcome $(6.2 \%$ vs $1.5 \%$, $\mathrm{p}<0.001)$ and better survival to discharge rate $(11.3 \%$ vs $3.3 \%, \mathrm{p}<0.001)$ than those transported P-CRC (Table 2).

Of the 1,681 patients who survived to admission, 1,025 (56.1\%) were admitted to DCRC. D-CRC provided higher proportions of post-resuscitation treatments, such as PCI (15.2\% vs 7.5\%, p<0.001), TTM (29.9\% vs $7.6 \%, \mathrm{p}<0.001)$, and extracorporeal membrane oxygenation $(5.5 \%$ vs $1.4 \%, \mathrm{p}<0.001)$ compared to P-CRC. Patients admitted to D-CRC showed better neurologic recovery ( $24.3 \%$ vs $13.1 \%, \mathrm{p}<0.001)$ and survival to discharge $(44.0 \%$ vs $29.7 \%, \mathrm{p}<0.001)$ than those admitted to P-CRC (Table 3).

Table 1. Result of Hospital Assessment Survey and classification of CRCs by result

\begin{tabular}{|c|c|c|c|}
\hline \multicolumn{2}{|l|}{ Hospital Assessment Survey } & \multirow{2}{*}{$\begin{array}{l}\text { n } \\
77\end{array}$} & \multirow{2}{*}{$\begin{array}{l}\% \\
100.0\end{array}$} \\
\hline Questionnaire & & & \\
\hline \multirow[t]{5}{*}{ 1. Level of your emergency department at your medical facility? } & Regional EMC & 4 & 4.4 \\
\hline & Local EMC & 26 & 26.3 \\
\hline & Local ED & 33 & 33.4 \\
\hline & Non-ED facility & 14 & 14.2 \\
\hline & NA & 13 & 13.1 \\
\hline \multirow[t]{2}{*}{ 2. Does your medical facility usually admit or transfer post-ROSC patients? } & Admit & 50 & 64.9 \\
\hline & Transfer & 27 & 35.1 \\
\hline \multirow[t]{2}{*}{ 3.Does your medical facility have a standard resuscitation protocol for OHCA at ED? } & Yes & 67 & 87.0 \\
\hline & No & 10 & 13.0 \\
\hline \multirow{2}{*}{$\begin{array}{l}\text { 4. Does your medical facility have a standard inter-hospital transfer protocol for post- } \\
\text { ROSC patients? }\end{array}$} & Yes & 48 & 62.3 \\
\hline & No & 29 & 37.7 \\
\hline \multirow{2}{*}{$\begin{array}{l}\text { 5. Does your medical facility have any device and manpower to provide therapeutic hypo- } \\
\text { thermia to post-ROSC patients? }\end{array}$} & Yes & 22 & 28.6 \\
\hline & No & 55 & 71.4 \\
\hline \multirow{2}{*}{$\begin{array}{l}\text { 6. Does your medical facility have a standard protocol for providing therapeutic hypother- } \\
\text { mia? }\end{array}$} & Yes & 18 & 23.4 \\
\hline & No & 59 & 76.6 \\
\hline \multirow[t]{2}{*}{ 7. Is PCI available $24 / 7$ at your medical facility? } & Yes & 29 & 37.7 \\
\hline & No & 48 & 62.3 \\
\hline \multicolumn{2}{|l|}{ 8. Does your medical facility have a specialized registration system for OHCA resuscitationYes } & 23 & 29.9 \\
\hline and treatment? & No & 54 & 70.1 \\
\hline \multirow{2}{*}{$\begin{array}{l}\text { 9. Does your medical facility perform quality management of OHCA resuscitation and } \\
\text { treatment? }\end{array}$} & Yes & 27 & 35.1 \\
\hline & No & 50 & 64.9 \\
\hline \multirow{2}{*}{$\begin{array}{l}\text { 10. Does your medical facility have a standard termination of resuscitation rule (or proto- } \\
\text { col)? }\end{array}$} & Yes & 36 & 46.8 \\
\hline & No & 41 & 53.3 \\
\hline \multirow[t]{2}{*}{ 11. Is EEG available to post-resuscitation patients at your medical facility? } & Yes & 35 & 45.5 \\
\hline & No & 42 & 54.6 \\
\hline \multirow[t]{2}{*}{ 12. Is confirmation of brain death possible at your medical facility? } & Yes & 32 & 41.6 \\
\hline & No & 45 & 58.4 \\
\hline \multirow{2}{*}{$\begin{array}{l}\text { 13. Is rehabilitation program available to post-resuscitation patients at your medical facil- } \\
\text { ity? }\end{array}$} & Yes & 38 & 49.4 \\
\hline & No & 39 & 50.7 \\
\hline \multirow[t]{2}{*}{ 14. Does your medical facility provide CPR education to the community? } & Yes & 54 & 70.1 \\
\hline & No & 23 & 29.9 \\
\hline Cardiac Resuscitation Center Classification & Medical facilities & \multicolumn{2}{|c|}{ OHCA (2012-2014) } \\
\hline Total number of hospitals that participated in the survey & 77 & \multicolumn{2}{|c|}{$9,912(100 \%)$} \\
\hline D-CRC & 15 & \multicolumn{2}{|c|}{$4,036(40.7 \%)$} \\
\hline P-CRC & 62 & \multicolumn{2}{|c|}{$5,876(59.3 \%)$} \\
\hline
\end{tabular}

CPR, cardiopulmonary resuscitation; CRC, cardiac resuscitation center; D-CRC, definite cardiac resuscitation center; ED, emergency department; EEG, electroencephalography; EMC, emergency medical center; NA, no answer; OHCA, out-of-hospital cardiac arrest; PCI, percutaneous coronary intervention; P-CRC, primary cardiac resuscitation center; ROSC, return of spontaneous circulation. 
Table 2. Demographics and outcomes of all OHCA patients transported to CRCs

\begin{tabular}{|c|c|c|c|c|c|c|c|}
\hline \multirow[t]{2}{*}{ Risk factors } & \multicolumn{2}{|c|}{ All OHCA } & \multicolumn{2}{|c|}{ P-CRC } & \multicolumn{2}{|c|}{ D-CRC } & \multirow[t]{2}{*}{$\mathbf{P}^{\star}$} \\
\hline & $\mathbf{n}$ & $\%$ & $\mathbf{n}$ & $\%$ & $\mathbf{n}$ & $\%$ & \\
\hline All & 9,912 & 100.0 & 5,876 & 100.0 & 4,036 & 100.0 & \\
\hline Male & 6,286 & 63.4 & 3,654 & 62.2 & 2,632 & 65.2 & 0.002 \\
\hline \multicolumn{8}{|l|}{ Age } \\
\hline Median (IQR) & 71 & $55-80$ & 72 & $57-81$ & 68 & $54-79$ & $<0.001$ \\
\hline$<15$ years & 179 & 1.8 & 62 & 1.1 & 117 & 2.9 & $<0.001$ \\
\hline $15-64$ years & 3,684 & 37.2 & 2,058 & 35.0 & 1,626 & 40.3 & \\
\hline Older than 65 years & 6,049 & 61.0 & 3,756 & 63.9 & 2,293 & 56.8 & \\
\hline \multicolumn{8}{|l|}{ Pre-arrest comorbidities } \\
\hline Cardiac disease & 1,338 & 13.5 & 718 & 12.2 & 620 & 15.4 & $<0.001$ \\
\hline Hypertension & 3,250 & 32.8 & 1,759 & 29.9 & 1,491 & 36.9 & $<0.001$ \\
\hline Diabetes mellitus & 2,191 & 22.1 & 1,209 & 20.6 & 982 & 24.3 & $<0.001$ \\
\hline Cardiac arrest location & & & & & & & $<0.001$ \\
\hline Public & 1,579 & 15.9 & 875 & 14.9 & 704 & 17.4 & \\
\hline Private & 1,644 & 16.6 & 1,064 & 18.1 & 580 & 14.4 & \\
\hline Unknown & 6,689 & 67.5 & 3,937 & 67.0 & 2,752 & 68.2 & \\
\hline Witness & 4,827 & 48.7 & 2,684 & 45.7 & 2,143 & 53.1 & $<0.001$ \\
\hline \multicolumn{3}{|l|}{ Who witnessed or found } & & & & & $<0.001$ \\
\hline EMT & 1,139 & 11.5 & 598 & 10.2 & 541 & 13.4 & \\
\hline Layperson & 6,764 & 68.2 & 3,977 & 67.7 & 2,787 & 69.1 & \\
\hline Unknown & 2,009 & 20.3 & 1,301 & 22.1 & 708 & 17.5 & \\
\hline Bystander CPR & 4,743 & 47.9 & 2,703 & 46.0 & 2,040 & 50.6 & $<0.001$ \\
\hline Initial EMS ECG & & & & & & & $<0.001$ \\
\hline Shockable & 1,321 & 13.3 & 639 & 10.9 & 682 & 16.9 & \\
\hline Non-shockable & 8,222 & 83.0 & 5,019 & 85.4 & 3,203 & 79.4 & \\
\hline Unknown & 369 & 3.7 & 218 & 3.7 & 151 & 3.7 & \\
\hline \multicolumn{8}{|l|}{ EMS response intv. } \\
\hline Median (IQR) & 8 & $6-10.5$ & 8 & $6-11$ & 7 & $6-10$ & $<0.001$ \\
\hline$<4$ minutes & 426 & 4.3 & 241 & 4.1 & 185 & 4.6 & $<0.001$ \\
\hline $4-8$ minutes & 4,262 & 43.0 & 2,379 & 40.5 & 1,883 & 46.7 & \\
\hline Over 8 minutes & 5,224 & 52.7 & 3,256 & 55.4 & 1,968 & 48.8 & \\
\hline \multicolumn{8}{|l|}{ Scene resuscitation intv. } \\
\hline Median (IQR) & 8 & $5-12$ & 8 & $5-11$ & 9 & $6-12$ & $<0.001$ \\
\hline$<5$ minutes & 1,608 & 16.2 & 978 & 16.6 & 630 & 15.6 & $<0.001$ \\
\hline 5-15 minutes & 6,973 & 70.4 & 4,209 & 71.6 & 2,764 & 68.5 & \\
\hline Over 15 minutes & 1,331 & 13.4 & 689 & 11.7 & 642 & 15.9 & \\
\hline \multicolumn{8}{|l|}{ Patient transport intv. } \\
\hline Median (IQR) & 7 & $5-10$ & 6 & $4-10$ & 7 & $5-10$ & $<0.001$ \\
\hline$<15$ minutes & 8,702 & 87.8 & 5,214 & 88.7 & 3,488 & 86.4 & $<0.001$ \\
\hline 15-30 minutes & 1,047 & 10.6 & 617 & 10.5 & 430 & 10.7 & \\
\hline Over 30 minutes & 163 & 1.6 & 45 & 0.8 & 118 & 2.9 & \\
\hline Pre-hospital ROSC & 569 & 5.7 & 163 & 2.8 & 406 & 10.1 & $<0.001$ \\
\hline Survival to admission & 1,681 & 17.0 & 656 & 11.2 & 1,025 & 25.4 & $<0.001$ \\
\hline Survival to discharge & 651 & 6.6 & 196 & 3.3 & 455 & 11.3 & $<0.001$ \\
\hline Good neurology & 338 & 3.4 & 87 & 1.5 & 251 & 6.2 & $<0.001$ \\
\hline
\end{tabular}

CPR, cardiopulmonary resuscitation; CRC, cardiac resuscitation center; D-CRC, definite cardiac resuscitation center; ECG, electrocardiography; ED, emergency department; EEG, electroencephalography; EMC, emergency medical center; EMS, emergency medical service; EMT, emergency medical technician; IQR, interquartile range; NA, no answer; OHCA, out-of-hospital cardiac arrest; PCI, percutaneous coronary intervention; P-CRC, primary cardiac resuscitation center; ROSC, return of spontaneous circulation.

* P-values were calculated using the Wilcoxon-rank sum test and chi-square test, as appropriate. 
Table 3. Demographics and outcomes of admitted OHCA patients transported to CRCs

\begin{tabular}{|c|c|c|c|c|c|c|c|}
\hline \multirow[t]{2}{*}{ Risk factors } & \multicolumn{2}{|c|}{ All OHCA } & \multicolumn{2}{|c|}{ P-CRC } & \multicolumn{2}{|c|}{ D-CRC } & \multirow[t]{2}{*}{$\mathbf{P}^{\star}$} \\
\hline & $\mathrm{n}$ & $\%$ & $\mathrm{n}$ & $\%$ & $\mathrm{n}$ & $\%$ & \\
\hline All & 1,681 & 100.0 & 656 & 100.0 & 1,025 & 100.0 & \\
\hline Male & 1,149 & 68.4 & 432 & 65.9 & 717 & 70.0 & 0.078 \\
\hline \multicolumn{8}{|l|}{ Age } \\
\hline Median (IQR) & 61 & $50-74$ & 64 & $51-77$ & 60 & $49-73$ & $<0.001$ \\
\hline$<15$ years & 32 & 1.9 & 4 & 0.6 & 28 & 2.7 & $<0.001$ \\
\hline $15-64$ years & 905 & 53.8 & 327 & 49.9 & 578 & 56.4 & \\
\hline Older than 65 years & 744 & 44.3 & 325 & 49.5 & 419 & 40.9 & \\
\hline \multicolumn{8}{|l|}{ Pre-arrest comorbidities } \\
\hline Cardiac disease & 302 & 18.0 & 107 & 16.3 & 195 & 19.0 & 0.157 \\
\hline Hypertension & 672 & 40.0 & 247 & 37.7 & 425 & 41.5 & 0.119 \\
\hline Diabetes mellitus & 443 & 26.4 & 182 & 27.7 & 261 & 25.5 & 0.301 \\
\hline Location of cardiac arrest & & & & & & & $<0.001$ \\
\hline Public & 384 & 22.8 & 124 & 18.9 & 260 & 25.4 & \\
\hline Private & 363 & 21.6 & 174 & 26.5 & 189 & 18.4 & \\
\hline Unknown & 934 & 55.6 & 358 & 54.6 & 576 & 56.2 & \\
\hline Witness & 1,134 & 67.5 & 432 & 65.9 & 702 & 68.5 & 0.127 \\
\hline \multicolumn{8}{|l|}{ Who witnessed or found } \\
\hline EMT & 347 & 20.6 & 137 & 20.9 & 210 & 20.5 & 0.069 \\
\hline Layperson & 1,036 & 61.6 & 386 & 58.8 & 650 & 63.4 & \\
\hline Unknown & 298 & 17.7 & 133 & 20.3 & 165 & 16.1 & \\
\hline Bystander CPR & 937 & 55.7 & 358 & 54.6 & 579 & 56.5 & 0.665 \\
\hline Initial EMS ECG & & & & & & & 0.014 \\
\hline Shockable & 509 & 30.3 & 175 & 26.7 & 334 & 32.6 & \\
\hline Non-shockable & 1,100 & 65.4 & 457 & 69.7 & 643 & 62.7 & \\
\hline Unknown & 72 & 4.3 & 24 & 3.7 & 48 & 4.7 & \\
\hline EMS response intv. & & & & & & & 0.991 \\
\hline Median (IQR) & 7 & $5-9$ & 7 & $5-9.5$ & 7 & $5-9$ & 0.546 \\
\hline$<4$ minutes & 90 & 5.4 & 39 & 6.0 & 51 & 5.0 & \\
\hline 4-8 minutes & 850 & 50.6 & 323 & 49.2 & 527 & 51.4 & \\
\hline Over 8 minutes & 741 & 44.1 & 294 & 44.8 & 447 & 43.6 & \\
\hline Patient transport intv. & & & & & & & $<0.001$ \\
\hline Median (IQR) & 6 & $4-10$ & 5 & $4-8$ & 6 & $4-10$ & $<0.001$ \\
\hline$<15$ minutes & 1,465 & 87.2 & 613 & 93.5 & 852 & 83.1 & \\
\hline 15-30 minutes & 145 & 8.6 & 40 & 6.1 & 105 & 10.2 & \\
\hline Over 30 minutes & 71 & 4.2 & 3 & 0.5 & 68 & 6.6 & \\
\hline \multicolumn{8}{|l|}{ Post-resuscitation care } \\
\hline PCI & 205 & 12.2 & 49 & 7.5 & 156 & 15.2 & $<0.001$ \\
\hline TTM & 356 & 21.2 & 50 & 7.6 & 306 & 29.9 & $<0.001$ \\
\hline ECMO & 65 & 3.9 & 9 & 1.4 & 56 & 5.5 & $<0.001$ \\
\hline Pre-hospital ROSC & 453 & 27.0 & 114 & 17.4 & 339 & 33.1 & $<0.001$ \\
\hline Survival to discharge & 646 & 38.4 & 195 & 29.7 & 451 & 44.0 & $<0.001$ \\
\hline Good neurology & 335 & 19.9 & 86 & 13.1 & 249 & 24.3 & $<0.001$ \\
\hline
\end{tabular}

CPR, cardiopulmonary resuscitation; CRC, cardiac resuscitation center; D-CRC, definite cardiac resuscitation center; ECG, electrocardiography; ECMO, extracorporeal membrane oxygenation circulation, ED, emergency department; EEG, electroencephalography; EMC, emergency medical center; EMS, emergency medical service; EMT, emergency medical technician; IQR, interquartile range; NA, no answer; OHCA, out-of-hospital cardiac arrest; PCI, percutaneous coronary intervention; P-CRC, primary cardiac resuscitation center; ROSC, return of spontaneous; TTM, target temperature management.

${ }^{\star} \mathrm{P}$-values were calculated using the Wilcoxon-rank sum test and chi-square test, as appropriate. 
Table 4. Effect of CRC on the neurologic outcomes and survival to discharge

\begin{tabular}{|c|c|c|c|c|}
\hline & $\begin{array}{l}\text { Total } \\
\mathbf{n}\end{array}$ & $\begin{array}{l}\text { Outcome } \\
\text { n (\%) }\end{array}$ & $\begin{array}{l}\text { Unadjusted OR } \\
(95 \% \mathrm{CI})\end{array}$ & $\begin{array}{l}\text { Adjusted OR } \\
(95 \% \mathrm{CI})^{\mathrm{a}}\end{array}$ \\
\hline \multicolumn{2}{|c|}{ All OHCA } & \multicolumn{3}{|c|}{ Good neurology outcome } \\
\hline Total & 9,912 & $338(3.4 \%)$ & & \\
\hline P-CRC & 5,876 & $87(1.5 \%)$ & Reference & Reference \\
\hline \multirow[t]{2}{*}{ D-CRC } & 4,036 & $251(6.2 \%)$ & $4.41(3.45-5.65)$ & $2.10(1.51-2.92)$ \\
\hline & & \multicolumn{3}{|c|}{ Survival to discharge } \\
\hline Total & 9,912 & $651(6.6 \%)$ & & \\
\hline P-CRC & 5,876 & $196(3.3 \%)$ & Reference & Reference \\
\hline D-CRC & 4,036 & $455(11.3 \%)$ & $3.68(3.10-4.38)$ & $2.41(1.95-2.98)$ \\
\hline \multicolumn{2}{|c|}{ Survival to admitted OHCA } & \multicolumn{3}{|c|}{ Good neurology outcome } \\
\hline Total & 1,681 & $335(19.9 \%)$ & & \\
\hline P-CRC & 656 & $86(13.1 \%)$ & Reference & Reference \\
\hline \multirow[t]{2}{*}{ D-CRC } & 1,025 & $249(24.3 \%)$ & $2.13(1.63-2.78)$ & $1.48(1.02-2.14)$ \\
\hline & & \multicolumn{3}{|c|}{ Survival to discharge } \\
\hline Total & 1,681 & $646(38.4 \%)$ & & \\
\hline P-CRC & 656 & $195(29.7 \%)$ & Reference & Reference \\
\hline D-CRC & 1,025 & $451(44.0 \%)$ & $1.86(1.51-2.29)$ & $1.57(1.22-2.03)$ \\
\hline
\end{tabular}

CI, confidential interval; CRC, cardiac resuscitation center; D-CRC, definite cardiac resuscitation center, OHCA, out-of-hospital cardiac arrest; OR, odds ratio; P-CRC, primary cardiac resuscitation center.

a Adjusted for age, gender, medical conditions, initial ECG rhythm at scene, the presence of witness, provision of bystander CPR, EMS response interval, EMS scene resuscitation interval, patient transport interval, pre-hospital return of circulation

Among all OHCA patients, after adjusting for potential confounders, transportation to D-CRC was significantly associated with good neurologic recovery (aOR, 2.10; 95\% CI, 1.51-2.93) and survival to discharge (aOR, 4.41; 95\% CI, 3.45-5.65). For patients who survived to admission, D-CRC was significantly associated with better neurologic recovery than P-CRC (aOR, 1.48; 95\% CI, 1.02-2.14) (Table 4).

\section{DISCUSSION}

This is the first regional attempt to categorize cardiac arrest receiving hospitals based on their practices and resources and compare the outcomes between the two levels of cardiac arrest receiving hospitals. This population-based study demonstrated that OHCA patients transported to D-CRC had better neurologic outcome than those transported to P-CRC (Table 2). Patients who survived and were admitted to D-CRC showed better neurologic outcome than those admitted to P-CRC (Table 3 ). This result supports the hypothesis that comprehensive post-resuscitation care applied to successfully resuscitated patients results in a better neurologic outcome. Our result coincided with those of recent studies, which suggested that transferring OHCA patients to specialized cardiac arrest centers resulted in good neurologic recovery. $(8,10,12)$ From this study, we were

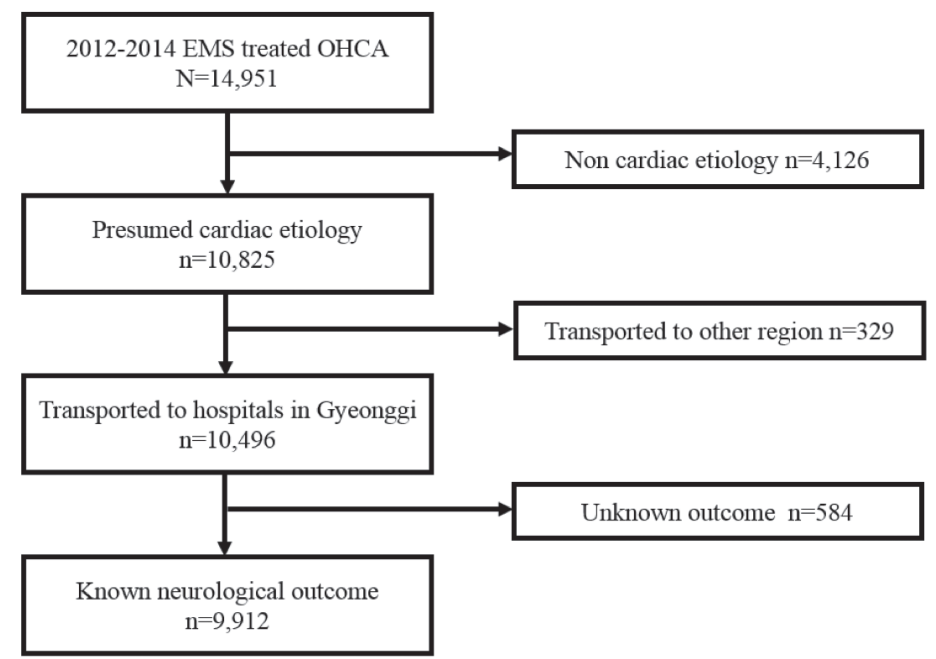

Figure 1. Flow chart of study population

EMS, emergency medical service; OHCA, out-of-hospital cardiac arrest.

able to assess the distribution of hospital resources and OHCA results based on the distribution of our community. This result will be helpful in establishing the transport protocol of EMS and inter-hospital transfer strategy after OHCA.

The result of this study suggests that it is desirable for the prognosis of OHCA patients to be transported to D-CRC. However, in a large district like Gyeonggi province, direct transfer of OHCA patients to
D-CRC may result in long-distance transportation with resuscitation performed at the back of the moving ambulance. Many studies have demonstrated that the quality of CPR is suboptimal during ambulance transportation. Korean EMTs are legally restricted from performing advanced skills such as endotracheal intubation or intravenous drug infusion. The ambulance crew is composed of not more than three members. Therefore, to overcome 
these legal and resource barriers, instead of transporting OHCA patients to DCRC regardless of transport interval, it is important to provide high-quality resuscitation at P-CRC and transfer patients safely to D-CRC as soon as they achieve ROSC. The American Heart Association proposed 2 levels of CRCs and provided the criteria for each center. Arizona established a statewide network of hospitals classified by the government as 'cardiac receiving centers' or 'cardiac referral centers'. $(8,10)$ Although the criteria should be reevaluated periodically, the basic concept of a regional system of care is focused on providing specialized post-resuscitation care in selected hospitals and transferring post-ROSC patients to these hospitals as appropriate. (6)

A safe inter-hospital transfer protocol between P-CRC and D-CRC is also essential to the regionalization of OHCA care. A previous study reported that therapeutic hypothermia had a less beneficial effect on the neurologic recovery of patients who arrived via inter-hospital transfer than those who were directly transported to the hospital. (13) Other literature reported that good neurologic recovery was less frequently observed among patients who experienced any events during the transfer. (14) To date, a successful inter-hospital transfer protocol for post-resuscitation care has been integrated into an existing regional system of care for STEMI. (15) No study was able to define the role of secondary transfer to a regional center after initial care at primary care hospitals. Nevertheless, inter-hospital transfer is an inevitable procedure in operating a re- gionalization strategy especially with the EMS system at an intermediate level. Considering that this is the first study that attempts to suggest a regionalization model in Korea, developing a safe inter-hospital transfer protocol based on the current resources would be necessary.

Lastly, optimization of a regionalization strategy in communities must be implemented. The interval between EMS arrival on the scene and transport to a D-CRC is unduly long in rural and suburban areas of the Gyeonggi province. Although observational studies suggested that the duration of transport to the hospital was not associated with patient outcomes, this negative association was only observed among OHCA patients successfully resuscitated on-scene. $(16,17)$ To date, no study has been able to provide a safe transport interval for OHCA patients who failed to achieve ROSC in the field. A priori categorization, verification, and designation of CRCs based on their actual practice performances should be done within the community. High-quality resuscitation at P-CRCs followed by a safe transfer to DCRCs to provide multidisciplinary postresuscitation care would enhance the survival of OHCA patients.

\section{LIMITATIONS}

This study had several limitations. First, all patients with unknown final outcome were excluded from the study. Second, the year of survey (2015) and OHCA occurrence (2012-2014) differed. However, the list of facilities for the survey was selected if they received an OHCA patient during 2013-2014; thus, it would not have affected the result. Third, post-resuscitation care, such as PCI timing or TTM protocol, was not standardized among the CRCs. Although patient care policy may differ between CRCs, this would increase the generalizability of our study findings. Fourth, similar to other observational studies, the unmeasured bias would have influenced the study. Lastly, the result of this study can only be implemented in an EMS system where field termination of OHCA is legally not allowed.

\section{CONCLUSION}

Through this study, we were able to categorize CRCs according to hospital resources in our region. After adjusting for potential confounders, D-CRC was independently associated with better neurologic outcome compared to P-CRC. This finding highlights an important opportunity to implement the regionalization strategy for OHCA in Gyeonggi province.

\section{ACKNOWLEDGMENT}

The OHCA data was supported by the National Emergency Management Agency of Korea and the Korean Centers for Disease Control and Prevention. The Hospital Assessment Survey was supported by the Health Policy Division of the Health and Welfare Bureau of Gyeonggi province.

\section{REFERENCES}

1. Hazinski MF, Nolan JP, Billi JE, Böttiger BW, Bossaert L, de Caen AR, et al. Part 1: Executive summary: 2010 International consensus on cardiopulmonary resuscitation and emergency cardiovascular care Ssience with treatment recommendations. Circulation 2010;122:S250-S605.

2. Lee SY, Song KJ, Shin SD, Ro YS, Hong KJ, Kim YT, et al. A disparity in outcomes of out-of-hospital cardiac arrest by community socioeconomic status: A ten-year observational study. Resuscitation 2018;126:130-6.

3. Bernard SA, Gray TW, Buist MD, Jones BM, Silvester W, Gutteridge G, et al. Treatment of comatose survivors of out-of-hospital cardiac arrest with induced hypothermia. N Engl J Med 2002;346:557-63.

4. Kim JY, Shin SD, Ro YS, Song KJ, Lee EJ, Park CB, et al. Post-resuscitation care and outcomes of out-of-hospital cardiac arrest: a nationwide propensity score-matching analysis. Resuscitation 2013;84:1068-77.

5. Testori C, Sterz F, Behringer W, Haugk M, Uray T, Zeiner A, et al. Mild therapeutic hypothermia is associated with favourable outcome in patients after cardiac arrest with non-shockable rhythms. Resuscitation 2011;82:1162-7.

6. Nichol G, Aufderheide TP, Eigel B, Neumar RW, Lurie KG, Bufalino VJ, et al. Regional systems of care for out-of-hospital cardiac arrest: A policy statement from the American Heart Association. Circulation 2010;121:709-29.

7. Bosson N, Kaji AH, Niemann JT, Eckstein M, Rashi P, Tadeo R, et al. Survival and neurologic outcome after out-of-hospital cardiac arrest: results one year after regionalization of post-cardiac arrest care in a large metropolitan area. Prehosp Emerg Care 2014;18:217-23.

8. Spaite DW, Bobrow BJ, Stolz U, Berg RA, Sanders AB, Kern KB, et al. Statewide regionalization of postarrest care for out-of-hospital cardiac arrest: association with survival and neurologic outcome. Ann Emerg Med 2014;64:496-506.

9. Perkins GD, Jacobs IG, Nadkarni VM, Berg RA, Bhanji F, Biarent D, et al. Cardiac arrest and cardiopulmonary resuscitation outcome reports: update of the Utstein Resuscitation Registry Templates for Out-of-Hospital Cardiac Arrest: a statement for healthcare profes- 
sionals from a task force of the International Liaison Committee on Resuscitation (American Heart Association, European Resuscitation Council, Australian and New Zealand Council on Resuscitation, Heart and Stroke Foundation of Canada, InterAmerican Heart Foundation, Resuscitation Council of Southern Africa, Resuscitation Council of Asia); and the American Heart Association Emergency Cardiovascular Care Committee and the Council on Cardiopulmonary, Critical Care, Perioperative and Resuscitation. Circulation 2015;132:1286-300.

10. Donnino MW, Rittenberger JC, Gaieski D, Cocchi MN, Giberson B, Peberdy MA, et al. The development and implementation of cardiac arrest centers. Resuscitation 2011;82:974-8.

11. Kern KB. Usefulness of cardiac arrest centers - extending lifesaving post-resuscitation therapies: the Arizona experience. Circ J 2015;79:1156-63.

12. Elmer J, Rittenberger JC, Coppler PJ, Guyette FX, Doshi AA, Callaway CW, et al. Long-term survival benefit from treatment at a specialty center after cardiac arrest. Resuscitation 2016;108:48-53.

13. Park JH, Ahn KO, Shin SD, Song KJ, Ro YS, Kim JY, et al. A multicentre observational study of inter-hospital transfer for postresuscitation care after out-of-hospital cardiac arrest. Resuscitation 2016;108:34-9.

14. Hartke A, Mumma BE, Rittenberger JC, Callaway CW, Guyette FX. Incidence of re-arrest and critical events during prolonged transport of post-cardiac arrest patients. Resuscitation 2010;81:938-42.

15. Mooney MR, Unger BT, Boland LL, Burke MN, Kebed KY, Graham KJ, et al. Therapeutic hypothermia after out-of-hospital cardiac arrest: evaluation of a regional system to increase access to cooling. Circulation 2011;124:206-14.

16. Spaite DW, Bobrow BJ, Vadeboncoeur TF, Chikani V, Clark L, Mullins T, et al. The impact of prehospital transport interval on survival in out-of-hospital cardiac arrest: implications for regionalization of post-resuscitation care. Resuscitation 2008;79:61-6.

17. Spaite DW, Stiell IG, Bobrow BJ, de Boer M, Maloney J, Denninghoff K, et al. Effect of transport interval on out-of-hospital cardiac arrest survival in the OPALS study: implications for triaging patients to specialized cardiac arrest centers. Ann Emerg Med 2009;54:248-55. 